\title{
Spectra of Ultrabroadband Squeezed Pulses and the Finite-Time Unruh-Davies Effect
}

\author{
T. L. M. Guedes, ${ }^{1,}{ }^{*}$ M. Kizmann, ${ }^{1}$ D. V. Seletskiy, ${ }^{1,2}$ A. Leitenstorfer, ${ }^{1}$ Guido Burkard, ${ }^{1}$ and A. S. Moskalenko ${ }^{1,3, \dagger}$ \\ ${ }^{1}$ Department of Physics and Center for Applied Photonics, University of Konstanz, D-78457 Konstanz, Germany \\ ${ }^{2}$ Department of Engineering Physics, Polytechnique Montréal, Montréal, H3T 1J4, Canada \\ ${ }^{3}$ Department of Physics, KAIST, Daejeon 34141, Republic of Korea
}

(Received 18 October 2018; published 8 February 2019)

\begin{abstract}
We study spectral properties of quantum radiation of ultimately short duration. In particular, we introduce a continuous multimode squeezing operator for the description of subcycle pulses of entangled photons generated by coherent-field driving in a thin nonlinear crystal with second-order susceptibility. We find the ultrabroadband spectra of the emitted quantum radiation perturbatively in the strength of the driving field. They can be related to the spectra expected in an Unruh-Davies experiment with a finite time of acceleration. In the time domain, we describe the corresponding behavior of the normally ordered electric field variance.
\end{abstract}

DOI: 10.1103/PhysRevLett.122.053604

Introduction.-In quantum optics, parametric downconversion (PDC) in nonlinear crystals (NXs) has been routinely used to generate pairs of monochromatic entangled photons [1,2]. The so-obtained squeezed states of light have found applications in a broad range of areas like gravitational wave detection $[3,4]$, quantum communication systems [5-7], and precision measurements [8,9]. The active interest in squeezed states can be mainly related to the fact that the variance of a given phase space quadrature (a quantum-optical analogue of a canonical variable) is lower for a squeezed state than for a coherent state, including the vacuum state itself. In order to fulfill Heisenberg's uncertainty principle, the variance of the conjugate quadrature exhibits the opposite behavior.

In recent years, theoretical and experimental efforts have been made to describe and generate multimode squeezed states [10-15]. Although they have already been experimentally realized by a number of groups $[12,15]$, most of the achievements so far are limited to squeezed states with relatively narrow spectra, where the central frequency approximation is still valid. New developments in ultrastable few-cycle laser sources and advanced detection techniques have paved the way for the generation of few-cycle pulses of midinfrared (MIR) squeezed light and the electro-optic detection of their electric field statistics with subcycle temporal resolution [16-18]. The subcycle features in the noise patterns of the generated quantum fields are due to the spatiotemporal modulation of the refractive index of the NX induced by the driving field [18]. This is analogous to a time-dependent metric for the space-time occupied by the electric field, which leads to photon creation in the perspective of a moving observer [19].

The spectral properties of ultrabroadband squeezed states are also of particular interest because they can elucidate connections between quantum gravitational effects and their tabletop optical analogues. A characteristic example is the Unruh-Davies effect [20,21], according to which an observer in a noninertial reference frame, moving with constant acceleration in the vacuum of an inertial reference frame, should detect thermal radiation. This phenomenon is closely related to the Hawking radiation believed to be emitted at the horizon of black holes [22].

The direct observation of these predictions is at the present time infeasible due to technological limitations, even if possibilities were indicated for particles in strong laser and Coulomb fields [23-25], and thus optical counterparts were proposed [26-31] as a means of studying the physics behind such effects. However, little attention has been paid to the effects of the unavoidably finite (and often short) duration of the effective acceleration experienced by either the light or the detector in the suggested experiments.

In this Letter, we first introduce a squeezing operator capable of describing the multimode states generated in a very thin NX with $\chi^{(2)}$ nonlinearity when a coherent ultrashort driving pulse is applied. The relevant experimental setup is schematically shown in Fig. 1(a). Because of the minute thickness of the crystal, phase matching can be assumed perfect. The driving pulse induces a nonlinear mixing cascade, with the PDC acting as a seed for the subsequent frequency-conversion processes. The superposition of these forms the structure of the emitted quantum field. Next, we study its spectral properties and confirm the ultrabroadband character of the generated pulses of squeezed light. Moreover, perturbative calculation of the time-dependent variance of the electric field operator links our work with related experimental results on subcycleresolved sampling of the electric field statistics of quantumoptical states [16-18]. Finally, we make a comparison of the obtained spectra for ultrabroadband squeezed pulses and thermal radiation, aiming to elucidate connections with 

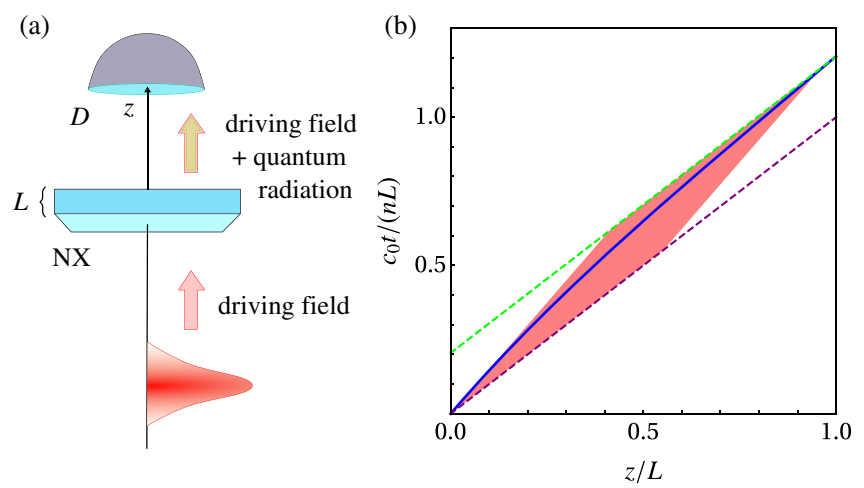

FIG. 1. (a) General sketch of the proposed experimental setup. The classical driving field propagates through a $\chi^{(2)}$ nonlinear crystal (NX) of small thickness $L$ and unperturbed refractive index $n$, generating ultrabroadband squeezed quantum light. The outgoing light is registered by the detector $D$. (b) World line of a plane wave mode of the quantum electric field within the NX with refractive index modulated by a half-cycle pulse (HCP). The trajectory (blue line) is given by Eq. (9) with $C_{1}=0$, $\alpha / n^{2}=0.49$, and $n \zeta=12$. The dotted purple straight line indicates the trajectory of light in the absence of nonlinear effects. After the acceleration has mostly ceased, the world line approaches the dotted green line parallel to the purple one. The process of acceleration is confined to a diamondlike space-time zone (light red parallelogram) of dimensions defined by the duration of the driving transient.

the Unruh-Davies radiation. It turns out that the limited lifetime of the refractive index perturbation in the crystal results in spectra with exponentially decaying highfrequency tails, which depend on the duration of the perturbation. This result can be related to the diamond temperature [32,33] derived for the Unruh-Davies effect when the observer follows an accelerated trajectory during a finite time interval. In our treatment, however, it is the observed incoming vacuum state that undergoes an effective acceleration within a certain space-time zone. This is illustrated in Fig. 1(b) for a plane wave mode of a quantum field that simultaneously enters the crystal with the peak of the driving field.

Squeezing operator.-Considering previously proposed expressions for a multimode squeezing operator [11,34] and using the convention $\hat{a}(-\omega)=\hat{a}^{\dagger}(\omega)(\omega \in \mathbb{R})$ connecting creation and annihilation operators for positive and negative frequencies [35], we introduce the following ansatz for the form of the (continuous) multimode squeezing operator:

$\hat{S}[\xi]=\exp \left\{\frac{1}{2}\left[\xi_{\omega_{1}, \omega_{2}} \hat{a}\left(\omega_{1}\right) \hat{a}\left(\omega_{2}\right)-\xi_{\omega_{1}, \omega_{2}} \hat{a}^{\dagger}\left(\omega_{1}\right) \hat{a}^{\dagger}\left(\omega_{2}\right)\right]\right\}$.

Here and below, we employ a generalized Einstein convention, meaning that product terms are integrated from $-\infty$ to $\infty$ over all continuous variables with reoccurring natural indices whenever such indices appear. For the unitarity of the squeezing operator, the frequency-dependent squeezing parameter $\xi_{\omega, \omega^{\prime}}$ must satisfy $\xi_{\omega, \omega^{\prime}}=\xi_{\omega^{\prime}, \omega}$, since then $\hat{S}^{\dagger}[\xi]=$ $\hat{S}[-\xi]$ and, hence, $\hat{S}[\xi]^{\dagger} \hat{S}[\xi]=1$. Rewriting Eq. (1) solely in terms of positive frequencies would lead to four terms in the integrand of the exponent. Two of them correspond to parametric down-conversion, while the remaining two correspond to frequency conversion. Note that the introduction of negative frequencies allows us to compactify the equations, while also including contributions from frequencyconversion processes.

In order to calculate expectation values of operators for the states generated by Eq. (1), let us investigate how $\hat{a}$ and $\hat{a}^{\dagger}$ transform under $\hat{S}$. We utilize a common procedure in quantum optics [36] by introducing an auxiliary operator $\hat{G}[\tilde{z} ; \xi]=\hat{S}^{\tilde{z}}[\xi]$ for $\tilde{z} \in[0,1]$, which commutes with $\hat{S}[\xi]$. We then define $\hat{a}(\tilde{z} ; \omega)=\hat{G}^{\dagger}[\tilde{z} ; \xi] \hat{a}(\omega) \hat{G}[\tilde{z} ; \xi]$ so that $\hat{a}(0 ; \omega)=\hat{a}(\omega) \quad$ and $\quad \hat{a}(1 ; \omega)=\hat{a}^{\prime}(\omega)=\hat{S}^{\dagger}[\xi] \hat{a}(\omega) \hat{S}[\xi]$. The commutator can be calculated using $\left[\hat{a}(\omega), \hat{a}\left(\omega^{\prime}\right)\right]=$ $\delta\left(\omega+\omega^{\prime}\right)\left[\operatorname{sgn}(\omega)-\operatorname{sgn}\left(\omega^{\prime}\right)\right] / 2$ for any $\omega, \omega^{\prime} \in \mathbb{R}$. Differentiating $\hat{a}(\tilde{z} ; \omega)$ with respect to $\tilde{z}$ and inserting the expression for $\hat{G}[\tilde{z} ; \xi]$ leads to

$$
\begin{aligned}
\frac{\partial \hat{a}(\tilde{z} ; \omega)}{\partial \tilde{z}} & =\Xi_{\omega, \omega_{1}} \hat{a}\left(\tilde{z} ; \omega_{1}\right), \\
\Xi_{\omega, \omega^{\prime}} & =-\operatorname{sgn}(\omega)\left(\xi_{\omega,-\omega^{\prime}}-\xi_{\omega^{\prime},-\omega}^{*}\right) .
\end{aligned}
$$

This integro-differential operator equation can be solved perturbatively expanding in $\Xi$, resulting in the Bogoliubov transformation:

$$
\begin{aligned}
\hat{a}(\tilde{z} ; \omega) & =U_{\omega, \omega_{1}}(\tilde{z}) \hat{a}\left(\omega_{1}\right), \\
U_{\omega, \omega^{\prime}}(\tilde{z}) & =\delta\left(\omega-\omega^{\prime}\right)+\tilde{z} \Xi_{\omega, \omega^{\prime}}+\frac{\tilde{z}^{2}}{2 !} \Xi_{\omega, \omega_{1}} \Xi_{\omega_{1}, \omega^{\prime}}+\cdots .
\end{aligned}
$$

Equation (3) assures the relation $\hat{a}(\tilde{z} ;-\omega)=\hat{a}^{\dagger}(\tilde{z} ; \omega)$.

Spectra.-The squeezing process depends on the buildup of electric fields within the NX, which determine the squeezing parameter in Eq. (3). Such interacting fields $\hat{E}(z, t)$ propagating along the $z$ axis in the crystal [see Fig. 1(a)] can be expressed in terms of plane waves confined to a certain transverse area [17], $\hat{E}(z, t)=\hat{E}\left(z, \omega_{1}\right) \times$ $\exp \left[-i \omega_{1}\left(t-n z / c_{0}\right)\right]$. Here $c_{0}$ is the speed of light in free space and $n$ is the unperturbed refractive index of the medium. It can be found [18] that due to the $\chi^{(2)}$ interaction process a coherent MIR driving field of sufficiently large amplitude $E_{\mathrm{MIR}}=\langle\hat{E}\rangle$ with respect to the amplitude of vacuum fluctuations [16] modulates the quantum contribution $\delta \hat{E} \equiv \hat{E}-E_{\mathrm{MIR}}$ as

$$
\frac{\partial \delta \hat{E}(z ; \omega)}{\partial z}=\frac{i d \omega}{n c_{0}} E_{\mathrm{MIR}}^{*}\left(z ; \omega_{1}-\omega\right) \delta \hat{E}\left(z ; \omega_{1}\right) .
$$


Here $d$ is the effective nonlinear coefficient of the NX, considered to be dispersionless in the relevant frequency range.

We can now change the variable $z \rightarrow \tilde{z}=z / L$ in Eq. (4) and use [37] $\delta \hat{E}(z ; \omega)=i \operatorname{sgn}(\omega) \times$ $\sqrt{\left(\hbar|\omega| / 4 \pi \epsilon_{0} c_{0} n A\right)} \hat{a}(z ; \omega)$, where $A$ is the normalization area, $\hbar$ is the reduced Planck constant, and $\epsilon_{0}$ is the vacuum permittivity. If we consider that $E_{\mathrm{MIR}}(z ; \omega)$ does not change appreciably as a function of $z$, comparison of the result with Eq. (2) gives

$$
\Xi_{\omega, \omega^{\prime}}=i C \operatorname{sgn}\left(\omega^{\prime}\right) \sqrt{\left|\omega \omega^{\prime}\right|} E_{\mathrm{MIR}}\left(\omega-\omega^{\prime}\right),
$$

where $C=d L /\left(n c_{0}\right)$. Similar expressions have been used to describe independent frequency-conversion and PDC processes involving light pulses with a well-defined central frequency $[13,38]$. Furthermore, the considered phase matching conditions lead to spatially distinguished signal and idler pulses. In contrast, Eqs. (1) and (5) do not rely on the assumption of a bandwidth much smaller than the central frequency. There is also no separation in the propagation direction of the outgoing photons.

Using Eqs. (3) and (5) we calculate perturbatively in $d$ the expectation value of the spectral photon density (SPD) operator, $\hat{\rho}(\omega)=\hat{a}^{\dagger}(\omega) \hat{a}(\omega)$, for the state $\left|\{\xi\}_{\omega}\right\rangle=\hat{S}[\xi]|0\rangle$ resulting from the pulse-induced squeezing process [39]. It is instructive, however, to begin by analyzing continuous wave (cw) driving with frequency $\omega_{0}, E_{\mathrm{MIR}}(\tau)=$ $E_{0} e^{-i \omega_{0} \tau}+E_{0}^{*} e^{i \omega_{0} \tau}$. Because of the infinite duration of the $\mathrm{cw}$ field, the SPD diverges for any frequency of interest. In this case, the spectral photon flux density $\phi(\omega)$ can be defined for a time interval $\Delta t$ and calculated [39], as is shown in Fig. 2(a). We see that PDC is maximally probable near the degeneracy point $\left(\omega \approx \omega_{0} / 2\right)$, while output at the drive frequency $\omega_{0}$ is absent. Additionally, higher-order contributions show that the photons generated by PDC can be up-converted to $3 \omega_{0} / 2$ by mixing with the coherent pump field.

Next, we study two cases of pulsed driving fields. Let us first consider an ideal half-cycle pulse (HCP) of light with temporal profile $E_{\mathrm{MIR}}(\tau)=E_{0} \operatorname{sech}(\Gamma \tau)$ and Fourier transform $E_{\mathrm{MIR}}(\omega)=\left(E_{0} / 2 \Gamma\right) \operatorname{sech}(\pi \omega / 2 \Gamma)$ [42]. In this case the SPD, $\rho(\omega)=\left\langle\{\xi\}_{\omega}|\hat{\rho}(\omega)|\{\xi\}_{\omega}\right\rangle$, reads

$$
\rho(\omega)=\frac{C^{2} E_{0}^{2}}{\pi^{2}} \omega \ln \left(1+e^{-\pi \omega / \Gamma}\right) .
$$

For an ideal single-cycle pulse (SCP) of form $E_{\mathrm{MIR}}(\tau)=$ $-E_{0}(\Gamma \tau) \operatorname{sech}(\Gamma \tau)$, which corresponds to $E_{\mathrm{MIR}}(\omega)=$ $\left(\pi E_{0} / 4 i \Gamma\right) \operatorname{sech}(\pi \omega / 2 \Gamma) \tanh (\pi \omega / 2 \Gamma)$ in the frequency domain, we find

$\rho(\omega)=\frac{C^{2} E_{0}^{2}}{12} \omega\left[\ln \left(1+e^{-\pi \omega / \Gamma}\right)+\frac{1}{2} \operatorname{sech}^{2}\left(\frac{\pi \omega}{2 \Gamma}\right)\right]$.

Both Eqs. (6) and (7) show that for high frequencies the SPD falls off as $\exp (-\pi \omega / \Gamma)$; i.e., its exponential decay is
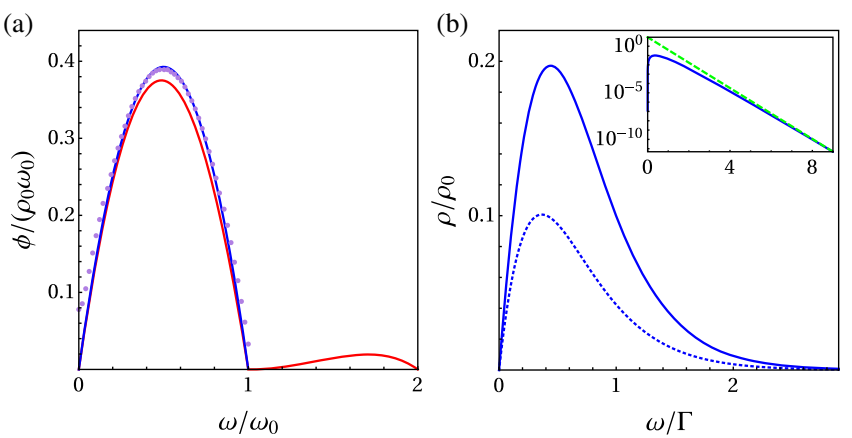

FIG. 2. (a) Normalized spectral photon flux density in the case of cw driving $\left(\rho_{0}=C^{2}\left|E_{0}\right|^{2} \Gamma / \pi^{2}, \Gamma=\omega_{0}\right.$ for $\left.\mathrm{cw}\right)$. Calculations including up to second-order (blue) and fourth-order (red) terms in $\alpha=d E_{0}$ have been included. The value of the factor $\pi^{4} \rho_{0} \omega_{0} / 4$ governing the smallness of the $\alpha^{4}$ term with respect to the $\alpha^{2}$ term is 0.02 . The dotted curve shows the average spectral photon flux density for a measurement over a finite time interval $\Delta t=N T$ with $N=50$, where $T=2 \pi / \omega_{0}$ is the period of the driving field. (b) Normalized spectral photon density (SPD) for the driving HCP (dotted blue line) and SCP (solid blue line) cases in the leading $\left(\alpha^{2}\right)$ order. The exponential behavior of the spectra can be better analyzed in a logarithmic plot, presented for the HCP case in the inset (same high-frequency behavior as for the SCP case). The SPD is shown in blue, while the asymptotic dotted straight line represents a fit of the form $A e^{-\pi(\omega / \Gamma)}$.

determined by the duration of the driving field $\Gamma^{-1}$ [see Fig. 2(b)].

Electric field variance.-Another insight into the generated quantum field is provided by its normally ordered variance (NOV), $V(\tau)$, which can be calculated as $V(\tau)=$ $\left\langle\{\xi\}_{\omega}\left|:[\delta \hat{E}(\tau)]^{2}:\right|\{\xi\}_{\omega}\right\rangle$, since $\left\langle\{\xi\}_{\omega}|: \delta \hat{E}(\tau):|\{\xi\}_{\omega}\right\rangle=0$. Here $: \hat{O}:$ denotes normal ordering for an operator $\hat{O}$ [36]. For the first-order term in the squeezing strength $r=|\alpha| \zeta / n$ $\left(\alpha=d E_{0}, \zeta=\Gamma L / c_{0}\right)$, we obtain [39]

$$
V^{(1)}(\tau)=\frac{\hbar C}{24 \pi \epsilon_{0} c_{0} n A} \frac{\partial^{3} E_{\mathrm{MIR}}(\tau)}{\partial \tau^{3}} .
$$

The corresponding second-order term, $V^{(2)}(\tau)$, was also calculated (for details, see Ref. [39]). The resulting temporal traces are shown in Fig. 3 for the considered $E_{\text {MIR }}$ shapes with driving field strengths within the validity of our perturbation approach.

The dynamics of the NOV is accessible via quantum electro-optic sampling [16-18] when the time resolution and sensitivity are high enough [43]. Within the range of validity of our perturbation theory, both the NOV and the SPD are interrelated via the shape of $E_{\mathrm{MIR}}$, motivating corresponding experiments.

Analogue gravity and world lines of light.-The quantum properties of the generated light are determined by the effectively curved space-time that the light modes experience while traveling through the NX, dressed by the input driving field. The metrics of such space-time can be 


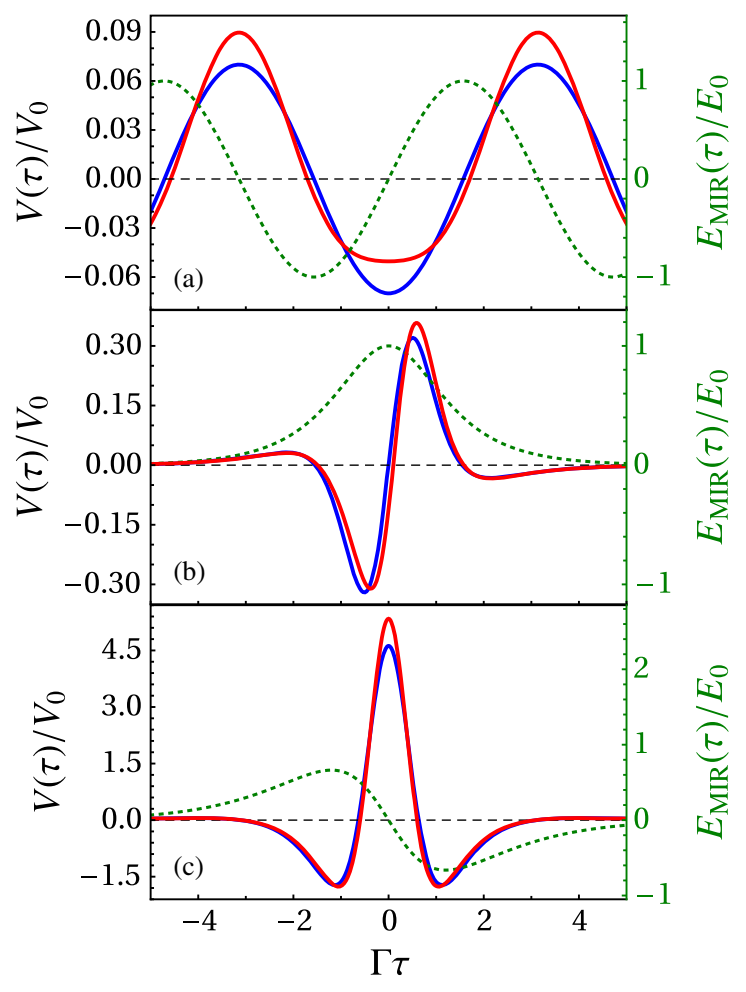

FIG. 3. Dynamics of the normally ordered variance (NOV), $V(\tau)$, of the emitted quantum electric field for (a) cw, (b) HCP, and (c) SCP driving (dotted green line). Contributions up to the first $V^{(1)}(\tau)$ (blue line) and the second $V^{(1)}(\tau)+V^{(2)}(\tau)$ (red line) order in the squeezing strength $r$ are shown. The NOV is normalized by $V_{0}=\hbar \Gamma^{2} /\left(24 \pi \epsilon_{0} c_{0} n A\right)$, while time is normalized by $\Gamma$ ( $\Gamma=\omega_{0}$ for cw driving). $r=0.07$ for (a), 0.21 for (b), and 1.54 for $(\mathrm{c})$.

extracted from the dispersion relation for the propagating quantum electric field $[44,45]$. This fact allows us to derive the null geodesic equations [46] for the respective modes (for details, see Ref. [39]). The world lines follow the equations

$$
\begin{gathered}
\frac{\alpha \zeta}{n} \frac{z}{L}-\sinh \left(\frac{\zeta}{L}\left(c_{0} t-n z\right)\right)=C_{1}, \\
\frac{\alpha \zeta}{n} \frac{z}{L}+\operatorname{Chi}\left(\left|\frac{\zeta}{L}\left(c_{0} t-n z\right)\right|\right)=C_{2},
\end{gathered}
$$

for the $\mathrm{HCP}$ and SCP driving cases, respectively. The constants $C_{1}$ and $C_{2}$ in these equations define the distance of a propagating wave front relative to the center of the driving field at the entrance of the crystal. $\alpha=d E_{0}$ gives the strength of the nonlinear perturbation, $\zeta=\Gamma L / c_{0}$ determines the spatial extension of the curvature (i.e., acceleration) relative to the length of the crystal, and $\operatorname{Chi}(x)$ is the hyperbolic cosine integral function [47]. The world lines for several values of $C_{1}$ and $C_{2}$ are shown in Fig. 4 alongside a projection of the normalized driving fields. The acceleration of the modes is confined to finite
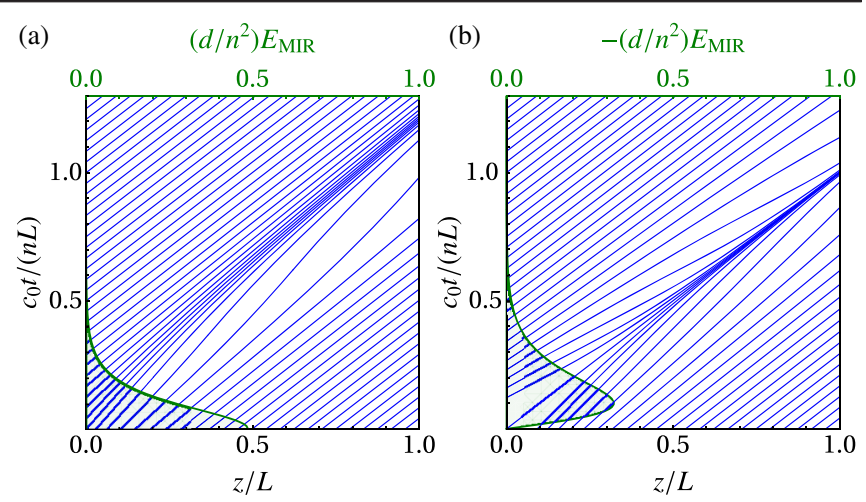

FIG. 4. World lines of the modes of quantum light propagating through the NX for HCP (a) and SCP (b) driving. Each world line (blue) is defined by its initial condition, which is given by a certain event at the boundary of the crystal and correspondingly by the amplitude of the driving field (green line) at this event. Here $\alpha / n^{2}=0.49$ and $n \zeta=12$ (see Ref. [39]).

regions of space-time. Moreover, the density of world lines projected along a line perpendicular to the light rays $t-n z / c_{0}=\tau=$ const determines the effective change in the flow of time and thereby is connected with the temporal profiles of the detected variance [43], as can be comprehended through comparison between Figs. 3(b) and 3(c) and Figs. 4(a) and 4(b). The evolution of the modes in the space-time curved due to a spatiotemporal varying refractive index leads to the generation of quantum radiation. It is thus insightful to discuss our results in relation to one of the most well-known examples of the creation of quantum light from the vacuum through acceleration: the Unruh-Davies effect.

Unruh-Davies radiation and the diamond temperature.From Planck's law, the SPD of thermal radiation is dominated by $\exp \left[-\left(\hbar \omega / k_{B} T\right)\right]$ at large frequencies, with a decay dependent on the temperature $T$ ( $k_{B}$ is Boltzmann's constant). According to the Unruh-Davies effect [20,21], for the quantum radiation detected in the reference frame of a uniformly accelerated observer, moving in the vacuum of an inertial observer (Minkowski vacuum), the temperature is given by $T_{U}=\hbar a /\left(2 \pi k_{B} c_{0}\right)$. Here $a$ is the acceleration measured in the accelerated observer's reference frame.

In the context of an optical analogue of the UnruhDavies effect, the detector remains at rest while the light follows an accelerated trajectory within a nonlinear material with time-varying refractive index. If one employs a crystal with $\chi^{(2)}$ nonlinearity as such a material, the refractive index can be modulated by a coherent driving [48] MIR field through the Pockels effect. To lowest order in $E_{\mathrm{MIR}}$ the acceleration of the quantum light modes within the crystal depends on its time derivative [27]. From Eqs. (6) and (7), however, it is possible to see that the exponential decay depends only on its duration $\Gamma^{-1}$.

Martinetti and Rovelli [32] considered the case of an observer with finite lifetime $\mathcal{T}$ uniformly accelerated in the 
vacuum of an inertial observer. In this case the Minkowski vacuum is observed as a thermal state with time-dependent temperature,

$T=T_{U} \varepsilon /\left[\sqrt{1+\varepsilon^{2}}-\sqrt{1+\varepsilon^{2} \tilde{t}^{2}}\right], \quad \varepsilon=2 T_{U} / T_{D}$,

where $\tilde{t}=2 t / \mathcal{T} \in(-1,1)$ is the normalized lab time and $T_{D}=2 \hbar /\left(\pi k_{B} \mathcal{T}\right)$. Since the observer's trajectory lies within a space-time diamond determined by $\mathcal{T}, T_{D}$ is termed the diamond's temperature. The minimal value $T_{\min }$ of $T$, in the middle of the observer's lifetime, should play the dominant role for the high-frequency tail of the emitted photon spectra. For sufficiently large lifetime or acceleration, $\varepsilon \gg 1$ and $T_{\min }$ coincides with the Unruh-Davies temperature $T_{U}$. In the opposite situation, $\varepsilon \ll 1$ and $T_{\min } \approx T_{D}$, given directly by the lifetime of the accelerated observer. This result was reinforced through analysis of a two-level detector model with a properly scaled Hamiltonian, which for a finite measurement time reveals that the detected temperature should be $T_{D}$ [33].

The analysis of the present work holds when $\varepsilon$ is small enough [39]. The spectra of the outgoing quantum light in our analogue optical system should decay as $\exp \left[-\left(\hbar \omega / k_{B} T\right)\right]$ with a temperature related to the duration of $E_{\mathrm{MIR}}$, since it dictates the duration of the acceleration of light within the NX. This result is reflected in Eqs. (6) and (7) through the decay dependence on $\Gamma$. This can also be seen qualitatively in Figs. 1(b) and 4, where curved world lines are confined to certain space-time zones. The same does not happen in the case of a cw driving field, since the respective electric field has no defined time duration.

Conclusions.-We propose a generalized squeezing operator to describe ultrabroadband squeezed pulses generated in thin $\chi^{(2)} \mathrm{NXs}$ by MIR coherent driving fields. We analyze the spectral properties of these squeezed states for three different shapes of the driving field and connect these results to the time-dependent NOV of the electric field operator. Ultimately, we account for the finite duration of the driving MIR pulses and relate our results to the diamond's temperature in an Unruh-Davies-like effect with a finite lifetime for the observer.

Funding by the DFG within SFB 767 and the BadenWürttemberg Stiftung via the Eliteprogramme for Postdocs as well as by the LGFG PhD fellowship program and Young Scholar Fund of the University of Konstanz is gratefully acknowledged. The authors thank Takayuki Kurihara, Rudolf Haussmann, Philipp Sulzer, Maximilian Russ, and Matthew Brooks for the fruitful and elucidating discussions.

*thiago.lucena@uni-konstanz.de andrey.moskalenko@uni-konstanz.de

[1] L.-A. Wu, H. J. Kimble, J. L. Hall, and H. Wu, Phys. Rev. Lett. 57, 2520 (1986).
[2] P. G. Kwiat, K. Mattle, H. Weinfurter, A. Zeilinger, A. V. Sergienko, and Y. Shih, Phys. Rev. Lett. 75, 4337 (1995).

[3] C. M. Caves, Phys. Rev. D 23, 1693 (1981).

[4] J. Aasi et al., Nat. Photonics 7, 613 (2013).

[5] M. Hillery, Phys. Rev. A 61, 022309 (2000).

[6] J. L. O'Brien, A. Furusawa, and J. Vučković, Nat. Photonics 3, 687 (2009).

[7] N. Gisin, G. Ribordy, W. Tittel, and H. Zbinden, Rev. Mod. Phys. 74, 145 (2002).

[8] M. Xiao, L.-A. Wu, and H. J. Kimble, Phys. Rev. Lett. 59, 278 (1987).

[9] V. Giovannetti, S. Lloyd, and L. Maccone, Science 306, 1330 (2004).

[10] W. Wasilewski, A. I. Lvovsky, K. Banaszek, and C. Radzewicz, Phys. Rev. A 73, 063819 (2006).

[11] K. J. Blow, R. Loudon, S. J. D. Phoenix, and T. J. Shepherd, Phys. Rev. A 42, 4102 (1990).

[12] Y. Shaked, R. Pomerantz, R. Z. Vered, and A. Pe'er, New J. Phys. 16, 053012 (2014).

[13] A. Christ, B. Brecht, W. Mauerer, and C. Silberhorn, New J. Phys. 15, 053038 (2013).

[14] N. A. Ansari and V. I. Man'ko, Phys. Lett. A 223, 31 (1996).

[15] V. Ansari, G. Harder, M. Allgaier, B. Brecht, and C. Silberhorn, Phys. Rev. A 96, 063817 (2017).

[16] C. Riek, D. V. Seletskiy, A. S. Moskalenko, J. Schmidt, P. Krauspe, S. Eckart, S. Eggert, G. Burkard, and A. Leitenstorfer, Science 350, 420 (2015).

[17] A. S. Moskalenko, C. Riek, D. V. Seletskiy, G. Burkard, and A. Leitenstorfer, Phys. Rev. Lett. 115, 263601 (2015).

[18] C. Riek, P. Sulzer, M. Seeger, A. S. Moskalenko, G. Burkard, D. V. Seletskiy, and A. Leitenstorfer, Nature (London) 541, 376 (2017).

[19] N. D. Birrell and P. C. W. Davies, Quantum Fields in Curved Space (Cambridge University Press, New York, 1984).

[20] W. G. Unruh, Phys. Rev. D 14, 870 (1976).

[21] P. C. W. Davies, J. Phys. A 8, 609 (1975).

[22] S. W. Hawking, Commun. Math. Phys. 43, 199 (1975).

[23] P. Chen and T. Tajima, Phys. Rev. Lett. 83, 256 (1999).

[24] R. Schützhold, G. Schaller, and D. Habs, Phys. Rev. Lett. 97, 121302 (2006).

[25] M. Kalinski, Laser Phys. 15, 1367 (2005).

[26] E. Yablonovitch, Phys. Rev. Lett. 62, 1742 (1989).

[27] T. G. Philbin, C. Kuklewicz, S. Robertson, S. Hill, F. König, and U. Leonhardt, Science 319, 1367 (2008).

[28] F. Belgiorno, S. L. Cacciatori, M. Clerici, V. Gorini, G. Ortenzi, L. Rizzi, E. Rubino, V. G. Sala, and D. Faccio, Phys. Rev. Lett. 105, 203901 (2010).

[29] F. Belgiorno, S. L. Cacciatori, G. Ortenzi, L. Rizzi, V. Gorini, and D. Faccio, Phys. Rev. D 83, 024015 (2011).

[30] M. F. Linder, R. Schützhold, and W. G. Unruh, Phys. Rev. D 93, 104010 (2016).

[31] P. Chen and G. Mourou, Phys. Rev. Lett. 118, 045001 (2017).

[32] P. Martinetti and C. Rovelli, Classical Quantum Gravity 20, 4919 (2003).

[33] D. Su and T. C. Ralph, Phys. Rev. D 93, 044023 (2016).

[34] C. F. Lo and R. Sollie, Phys. Rev. A 47, 733 (1993).

[35] M. F. Maghrebi, R. Golestanian, and M. Kardar, Phys. Rev. A 88, 042509 (2013). 
[36] W. Vogel and D. Welsch, Quantum Optics (Wiley, Weinheim, 2006).

[37] R. Loudon, The Quantum Theory of Light (Oxford University Press, New York, 2000).

[38] K. E. Dorfman, F. Schlawin, and S. Mukamel, Rev. Mod. Phys. 88, 045008 (2016).

[39] See Supplemental Material at http://link.aps.org/ supplemental/10.1103/PhysRevLett.122.053604 which includes Refs. [40,41], for (1) derivation of expressions for the spectral photon density and the spectral photon flux density, (2) conditions for the convergence of the perturbative expansion, behavior of the high-frequency tail of the spectral photon density and role of the pulse shape, (3) derivation of the normally ordered electric field variance, (4) description of the world lines of propagating electric field modes in a nonlinear crystal.

[40] K. S. Thorne and R. D. Blandford, Modern Classical Physics: Optics, Fluids, Plasmas, Elasticity, Relativity, and Statistical Physics (Princeton University Press, Princeton, NJ, 2017).

[41] V. A. De Lorenci and R. Klippert, Phys. Rev. D 65, 064027 (2002).

[42] A. S. Moskalenko, Z.-G. Zhu, and J. Berakdar, Phys. Rep. 672, 1 (2017).

[43] M. Kizmann, T. L. M. Guedes, D. V. Seletskiy, A. S. Moskalenko, A. Leitenstorfer, and G. Burkard, arXiv:1807 .10519.

[44] M. Novello, V. A. De Lorenci, J. M. Salim, and R. Klippert, Phys. Rev. D 61, 045001 (2000).

[45] U. Leonhardt and P. Piwnicki, Phys. Rev. A 60, 4301 (1999).

[46] B. Schutz, A First Course in General Relativity (Cambridge University Press, New York, 2009).

[47] I. S. Gradshteyn and I. M. Ryzhik, Table of Integrals, Series, and Products (Academic Press, San Diego, 2014).

[48] R. J. Glauber, Phys. Rev. 131, 2766 (1963). 